\title{
Linguistic validation of the Diabetic foot ulcer scale short form (DFS-SF): a methodological approach
}

\author{
Validação linguística da forma abreviada da escala de úlcera do pé diabético \\ (DFS-SF)
}

Uiara Aline de Oliveira Kaizer (D), Neusa Maria Costa Alexandre (D), Maria Helena de Melo Lima (D), Roberta Cunha Matheus Rodrigues (D), Marilia Estêvam Cornélio (D), Thaís Moreira São-João (iD)

\begin{abstract}
Objective: to presenting the linguistic validation of the Diabetic Foot Ulcer Scale Short-Form (DFS-SF) among patients with ulcer diabetic foot (DFU) to Brazilian Portuguese and evaluate its content, practicability and acceptability. Methods and casuistry: a cross-sectional, methodological research, was conducted among 30 outpatients in follow-up for DF, assessed regarding QoL, sociodemographic and clinical data. International recommendations provided by the Mapi Research Trust on adaptation procedures were followed: the stages of Forward translation, Backward translation and the Cognitive interview were carried out. The Content Validity Index was calculated and a pre-test were gathered to produce a pre-final version. Practicability and acceptability were also assessed. Results: the semantic, idiomatic, cultural and conceptual equivalences between the linguistic validated and the original version were obtained. The DFS-SF was practical, well accepted and easy to understand. Conclusion: the linguistic validation process of the Brazilian version of the DFS-SF has been completed in accordance with internationally recommended standards. The instrument was easy to apply, to understand and presented short time for administration.
\end{abstract}

Keywords: Diabetes Mellitus, Diabetic foot, Quality of life, Validation study.

\section{RESUMO}

Objetivo: apresentar a validação linguística do DFS-SF entre pacientes com DFU para o português do Brasil e avaliar seu conteúdo, praticabilidade e aceitabilidade. Metodologia e casuística: foi realizada uma pesquisa metodológica de corte transversal entre 30 pacientes ambulatoriais em acompanhamento para DFU. Seguiram-se recomendações internacionais fornecidas pelo Mapi Research Trust sobre procedimentos de adaptação: tradução direta, tradução reversa e entrevista cognitiva. O Índice de Validade de Conteúdo foi calculado e um pré-teste foi coletado para produzir uma versão pré-final. A praticidade e aceitabilidade também foram avaliadas. Resultados: foram obtidas as equivalências semântica, idiomática, cultural e conceitual entre a versão linguística validada e a versão original. O DFS-SF foi prático, bem aceito e fácil de entender. Conclusão: O DFS-SF da versão brasileira foi concluído de acordo com os padrões internacionalmente recomendados, foi fácil de aplicar, entender e apresentou pouco tempo para administração.

Palavras-chave: Diabetes Mellitus, Pé diabético, Qualidade de vida, Estudos de validação.

University of Campinas. School of Nursing. Campinas (SP), Brazil. 


\section{INTRODUCTION}

The Diabetic Foot (DF) disease has been defined by the International Working Group on Diabetic Foot as the presence of "Infection, ulceration or destruction of tissues of the foot associated with neuropathy and/or peripheral artery disease in the lower extremity of people with (a history of) diabetes" ${ }^{\prime \prime}$.

The incidence of DF ulcers (DFU) in people with diabetes is estimated at between $19 \%$ and $34 \%^{2}$. It is also known that $25 \%$ to $56.5 \%$ of cases individuals with diabetes have foot injuries at some point in their life with high recurrence rates ${ }^{3}$ and mortality rates close to $40 \%{ }^{4}$.

DF disease is a condition that has serious consequences, given its great functional impairment that can lead to incapacitations, hospitalizations and even death. There is a need for prolonged rehabilitation and social support, causing a high cost in all countries ${ }^{5}$.

The DF disease has been understood as a serious public health problem; not only for the totality of cases it reaches, but also because it generates changes in both the functional, emotional, social, economic and labor aspects of the world scenario ${ }^{6-10}$.

These complications caused by DFU can impair autonomy, making patients dependent on family and friends; bringing them suffering due to the necessary changes in their lifestyle and the new need for help to carry out their activities of daily living ${ }^{11}$. In this sense, psychological and social problems can harm the patient and his/ hers family with respect to performing the necessary care for the treatment, compromising QoL ${ }^{5}$. Thus, health services should be aware of the psychosocial aspects, through the early identification of changes in QoL and the redirection of health care provided to people with DFU ${ }^{11}$.

Therefore, to evaluate the QoL of patients with DFU may be considered a challenging and complex task, which requires considerable care in choosing the appropriate instrument to measure it. In the world literature, nowadays the only specific instrument available to assess the impact of DFU ulcer on QoL is the Diabetic Foot Ulcer Scale (DFS) and its short form, the Diabetic Foot Ulcer
Scale Short-Form (DFS-SF). It is a specific instrument designed to measure the impact of DFU on the QoL of people with diabetes and is available for use in clinical trials to evaluate the benefits of treatment to ulcer healing among them, the improvement in QoL ${ }^{12,13}$.

DSF-SF was developed with focus groups with patients with diabetes to identify specific factors affecting QoL - such as foot ulcers and their treatment. From these concepts, it was validated containing the following domains: Leisure, Physical Health, Daily Life Activities, Emotions, Non-adherence, Family, Friends, Treatment, Satisfaction, Positive Attitude and Financial. DSF-SF demonstrated good measurement properties, with satisfactory evidences of reliability and validity ${ }^{12}$.

DFS-SF was created by analyzing data from a double-blind, placebo-controlled, randomized trial of the efficacy and safety of becaplermin (recombinant human platelet derived growth factor) in the treatment of DFU. Using these data, items that demonstrated poor psychometric properties were eliminated and exploratory factor analyzes were performed to develop a new scale. Finally, data from two additional clinical trials were used to assess the replicability of the new DFS-SF subscale structure ${ }^{12}$. Reliability and validity were evaluated in two clinical trials with diabetic patients with foot ulcers. A Reliability coefficient (Cronbach's alpha) was computed for each sub subscale in study 1 (326 individuals) and in study 2 (423 individuals). Satisfactory internal consistency with ranging from 0.80 up to $0.94^{12}$.

The original version of the DFS-SF was previously tested with the known gold standard for measuring health-related QoL, the Medical Outcomes Study 36-Item Short-Form Health Survey (SF-36). Therefore, correlations between SF36 and DFS-SF subscale scores were examined through a multitrait-multimethod matrix and ranged from 0.19 up to 0.62 , providing good evidence on DFS-SF's convergent and discriminant construct validity.

The results of the confirmatory factor analysis were inconsistent at the chi-square test, suggesting a lack of fit. Exploratory factor analysis results suggested that a six-factor solution is appropriate and high values for these coefficients 
suggest that the DFS-SF factor structure was highly stable. DFS-SF subscales were also examined for sensitivity to clinical condition and demographic characteristics, and although these constructs require a longer time frame to result in significant changes, good responsiveness was observed ${ }^{13}$.

This instrument was selected for the current research because it presented measurement properties with evidence of validity and reliability to measure the QoL of this population and the use of a shorter version may often be desirable, particularly when time is limited or respondent burden is of major concern. It is a multidimensional and easily understood instrument, which is not excessively long and may lead to sensitivity to change in clinical conditions. In addition, the instrument assesses QOL in different aspects such as leisure, physical health, dependence, daily life, emotions, worry and care for ulcers ${ }^{13}$.

This study aimed to provide a Brazilian version of the DFS-SF, evaluate content validity through expert committee review and check the practicability and acceptability of the Brazilian version.

\section{MATERIAL AND METHOD}

A methodological approach, based on the theoretical framework of cultural adaptation and validation was carried out ${ }^{14}$.

This was cross-sectional study. The sample was composed by thirty patients with type 1 or 2 diabetes mellitus (DM); who attended the following inclusion criteria: older than 18 years; with one up to two DFU of neuropathic or neuroischemic etiology, ranging in size from 0.5 to $30 \mathrm{~cm}^{2} 12$; able to read, understand and respond to a cognitive assessment; and Brazilian Portuguese speakers.

Participants were excluded if they presented healed ulcer; more than two ulcers; had ulcers resulting from causes others than DM; with connective tissue or ischemic diseases and those who were drug and alcohol dependent ${ }^{12}$.

Sample size for patient testing was considered according to international guidelines on cross cultural adaptation ${ }^{15}$, which recommends the enrollment of thirty participants for this stage of research.
For the data collection, sociodemographic characterization instruments were used, in addition to DFS-SF. Clinical and sociodemographic characterization for the characterization of the participants, an instrument based on that developed study validation of the Chinese diabetic foot ulcer scale developed by Huy et $a^{16}$ was used. The data evaluated are related to age, sex, educational level, family support, duration of ulcer and diabetes, ulcer condition (active or healed), type of ulcer and episode of ulcer recurrence.

The short version of the instrument Diabetic Foot Ulcer Scale-Short Form (DFS-SF) is composed of 29 items divided into six domains: Leisure, Physical Health, Dependence/Daily Life, Negative Emotions, Worried about ulcers/feet and Bothered by ulcer care ${ }^{12}$. Answers range from "Not at all" to "extremely", "none of the time" to "all of the time" and "not at all" to "great deal". For each question, there is a score from 1 (never) to 5 (all the time). The score of the items was based on a five-point Likert scale. The DFS-SF score is based on the sum of all items in each domain. All DFS scales are scored from zero up to 100, with higher scores indicating better QoL. Reliability and validity were assessed in two clinical trials ${ }^{13}$ with diabetic patients with foot ulcers. As to the evaluation of its reliability, DFS-SF demonstrated high internal consistency and satisfactory measurement stability (test-retest); satisfactory convergent and discriminant validity when compared to SF-36 and good responsiveness to clinical changes. Confirmatory and exploratory factorial analysis were also conducted ${ }^{13}$.

For the implementation of data collection tools the team responsible for contacting the author of the instrument of the original instrument, together with Mapi Research Trust, was consulted to provide the authorization for adaptation to the Portuguese language of Brazil. After obtaining the formal authorization for the linguistic validation of the DFS-SF to the Portuguese language of Brazil by MAPI, the instruction manual of the instrument was supplied along with its original version.

The data of the study were collected from the patients with type 1 or 2 diabetes mellitus who foot ulcer by the face-to-face interview method during the weekdays in the patient rooms at 
Diabetic Foot and Wound Outpatient Clinic. The patients were asked to fill out the data collection tools. Data collection time was between 7 min for each participant.

According to the MAPI recommendations, the linguistic validation of the DFS-SF consisted of 3 stages. The first was the Forward translation (included the production of a "reconciled" version), followed by the Backward translation and the third was the Cognitive interview (patient testing) (Figure 1).

Figure 1. Algorithm of the linguistic validation process (adapted from MAPI, 2016).

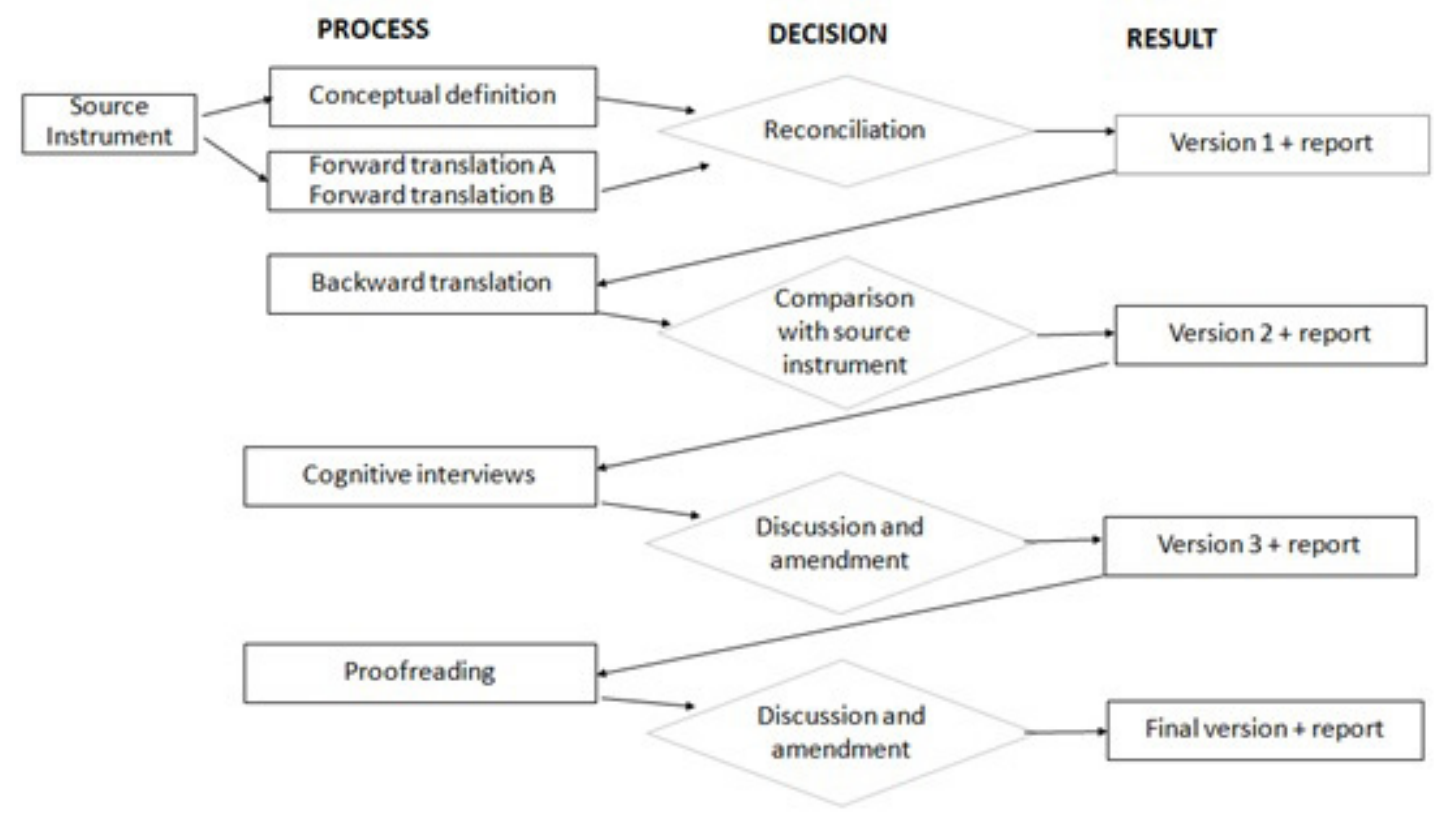

The steps of the linguistic validation process are described below.

The Forward Translation Step consisted of the translation of the questionnaire into the foreign language (Brazilian Portuguese, in this study). The original language in which the questionnaire was developed (English, for the DFS-SF) is called the source language. The foreign language which the questionnaire was translated into is called the target language. Therefore, the forward translation can be summed up as the passage from the source language into the target language. It required the recruitment of two local professional translators, which were two independent native target language speakers and bilingual in the source language; and also the involvement of the research coordinator, which was a nurse ${ }^{14}$.

This step's methodology involved the production of two forward translations - each of the translators produced an independent forward translation of the original items, instructions and response choices; and also the production of a pooled version (version 1) - both translators and the local coordinator discussed the translations and agreed on a reconciled version. The aim was a translation conceptually equivalent to the original questionnaire and with colloquial and easy to understand language by the target population.

This step also was based on the contact with the authors (MAPI) to discuss particular issues of the first version in the target language that had to be modified and the production of a report in English outlining the translation issues discussed and how the first version of the items, instructions and response choices were produced. As a result, this first step provided a first version of the DFS-SF together with a report ${ }^{14}$.

The Backward Translation Step consisted of the translation of the first version of the ques- 
tionnaire into the source language (English) and required the recruitment of a local professional translator, native speaker of the source language, bilingual in the target language ${ }^{14}$.

This step's methodology comprised the production of the backward version, by two other translators who translated the first version back into the source language. They had no access to the original version. This step also comprises the comparison of the backward version with the source instrument, done by the coordinator in order to detect any misunderstandings, mistranslations or inaccuracies in the first version of the questionnaire. This step resulted in changes to the first version, giving rise to the second version ${ }^{14}$.

Additionally to the steps recommended by the MAPI, other guidelines are internationally recognized - such as those proposed by Beaton and collaborators ${ }^{15}$. According to these authors, an expert committee should evaluate the pre-final version (before patient testing).

At this point, the produced version was analyzed by a committee of experts formed by bilingual people and specialists in the area of knowledge of the instrument. Eight bilingual judges participated in this committee, who met at least one of the following criteria: experience in cultural adaptation or in the Diabetes/ Diabetif Foot Disease field, research experience involving the application of measuring instruments and ability to recognize expressions in English and Portuguese languages.

The specialists received all versions of the instrument and each judge also received a specific questionnaire to carry out the evaluation ${ }^{17}$. They reviewed and compared all translated versions and changed or deleted inappropriate items until a final translation was obtained. The aim of this committee's work was to determine the semantic, linguistic, cultural and conceptual equivalence between the original questionnaire and the Portuguese version, in order to guarantee the understanding and cultural equivalence of the final version ${ }^{15}$.

These aspects were evaluated through the Content Validity Index (CVI). Among nurse researchers, CVI is the most widely reported measure of content validity. The panel of experts was asked to rate each scale item in terms of its relevance to the underlying construct. In our study, eight (8) judges assessed the instrument. By tradition, these item ratings are typically on a 4-point ordinal scale being the more frequent the following: $1=$ not relevant, $2=$ somewhat relevant, $3=$ quite relevant, $4=$ highly relevant. Then, for each item, the CVI was computed as the number of experts giving a rating of either 3 or, divided by the total number of experts. For example, an item that was rated as quite or highly relevant by four out of five judges would have an CVI of $0.80^{18}$.

The Patient Testing Step the aim of this phase was to test the translated questionnaire on the target population to determine whether it was acceptable, understood in the way it is supposed to and if the language used was simple and appropriate ${ }^{14}$.

To carry on this phase, the second version of the questionnaire (obtained after phase 2) was submitted to cognitive debriefing. The subjects recruited for the testing should all be native speakers of the target language. The comprehension test was performed through individual interviews, in which the interviewer inquired whether the participant had any difficulty in understanding the questionnaire and checked the participant's interpretation of all items. In case of any issue, the interviewer proposed or tested alternatives of translations, or asked the participant to propose alternatives ${ }^{14}$.

Individual interviews were conducted in a private room at the Clinic, by one of the trained researchers, live and in-person to catch the nuances and subtleties of responses. They were based at the cognitive debriefing method. Therefore, in the interview stage, respondents completed the questionnaire and then answer questions to explain their understanding of each question or item on the questionnaire. They restate in their own words what they think each item meant. Interviews were individually and qualitatively analyzed regarding its contents and each comment or suggestion made by participants was considered.

The aim of this phase The Proofreading Step was to avoid any typing, spelling or grammatical mistakes remain in the most recent target language version. The methodology of this phase 
consisted of proofreading of the third version of the questionnaire by a proofreader whose native language was the target language and who was proficient in English ${ }^{14}$.

This study was conducted at the Ambulatory of Wounds and Diabetic Foot located in a Municipal Polyclinic in a city in the interior of the state of São Paulo. Data were gathered between July and August 2017. All participants signed the consent form. The data were gathered by two trained researchers.

The collected data were entered into a spreadsheet in the software Microsoft Excel for Windows and then transferred to the Statistical Analysis System for Windows program, version 9.2 (Statistical Analysis System Institute Inc., Cary, NC, USA, 2008). Measures of position (mean, median, minimum and maximum) and dispersion (standard deviation) for sociodemographic and clinical characterization instrument data and for total score of $\mathrm{QOL}$ instruments. The Content Validity Index (CVI) calculation was used to evaluate the semantic-idiomatic, conceptual and cultural equivalence of the DFS-SF ${ }^{17,18}$. At the end of the patient testing step, the instrument was evaluated with regard to practicability and acceptability. Practicability refers to the instrument's application time length and acceptability is related to the respondents' understanding of the items composing the questionnaire, demonstrated by the percentage of unanswered items and by the proportion of patients who did not respond at all ${ }^{19}$.

All patients received information about the purpose of the study and it was guaranteed that the information would be confidential. The Helsinki Statement was followed. The study was approved by the local Research Ethics Committee (Document number 2,051,777 / 2017).

\section{RESULTS}

The cross cultural adaptation procedure of the DFS-SF followed the standards internationally recommended by MAPI and after the steps described, the translated version was obtained. The translators did not report difficulty translating the instrument except in the "Getting about" question (4. Because of your foot ulcer problems, have you felt: j) frustrated because you have difficulty in getting about), where one of the translators suggested that it meant "getting around" and not "continuing" as its original meaning.

After Backward Translation Step, the pre-final translated version was then submitted to the content validation step, through assessment by the experts committee for the evaluation of semantic-idiomatic, conceptual and cultural equivalences. The results showed CVI between 0.75 and 1 , which are satisfactory.

After the evaluation of the DFS-SF equivalences, minimal changes related to translated terms were suggested, such as replacing some words with synonyms for better understanding. Ex: the experts suggested to change the word "preferred" by "like", "odor" by "bad smell"; "Damages" for "losses"; for example. Suggestions were also made in order to maintain only one format in the sentences, such as standardize some terms in the singular or plural across the whole scale.

Adjustments have been suggested in some past tenses for better understanding, such as "has prevented" by "prevented"; "Had to depend" for "has depended" or "had to spend" for "having spent". It was also chosen to place some terms between relatives in an attempt to better explain some terms that respondents may not understand and keep the term in English in the sentence after the term translated into Portuguese, since its use in Brazilian culture is very common. Example: "Hobby (hobbies)".

The scale was divided into 42 item sin order to enable experts to evaluate each excerpt from the questionnaire. Therefore, as for the proportion of agreement among the experts regarding the analysis of the equivalences, of the 42 items only three obtained CVI smaller than 0.80 .

Item 1 (instrument title: Diabetic Foot Ulcer Scale-Short Form) obtained CVI of 0.75 in the semantic-idiomatic equivalence and some changes in the name of the scale were suggested as: "Scale for diabetic foot ulcer - abbreviated version", "Diabetic foot ulcer scale - abbreviated form" (Brazilian version). However, the authors decided to keep as "Brazilian Version of the Diabetic Foot Ulcer Scale-Short Form", as recommended internationally in order to elicitate finding this version across scientific publications, for example. 
In item 34 ("4. Because of your foot ulcer problems, have you felt: $j$ ) frustrated because you have difficulty in getting about"), both semantic-idiomatic, conceptual and cultural equivalences obtained CVI of 0.75 . The term "frustrated because you have difficulty in getting about", had some suggestions like: "frustrated by having difficulty in getting around". Another expert suggested to verify the meaning of the term "Getting about" with the authors of the original instrument and another expert considered that it was not clear what the item wanted to evaluate.

In item 37 ("5. Because of your foot ulcer problems, how often were you bothered by: a) having to keep the weight off your foot ulcer"), the semantic-idiomatic equivalence also obtained CVI of 0.75. The expression "having to keep your weight supported off the ulcer" caused disagreements among the experts and some suggestions were made such as: "not being able to support your weight on the foot that has the ulcer"; "Having to keep your weight supported from the foot that is with the ulcer"; "Having to keep your weight because of your foot ulcer". For a better understanding, authors have chosen to use "Have to keep your weight supported off the foot that has a ulcer."

A consensus face-to-face meeting was not necessary among the experts, because although five items had a CVI of less than 0.80 , the suggested modifications regarding order and agreement between words were followed and the authors, through consensus, made the adjustments and formulated the final version of the DFS-SF.

After the experts panel, the Patient Testing Step was conducted. Its sample $(n=30)$ consisted of a majority of men $(76.7 \%)$, mean age of 57.4 years, whites $(86.7 \%)$; living with partner $(33.3 \%)$ or with partner and sons $(33.3 \%)$; with more than eight years of study $(56.7 \%)$, were retired $(56.7 \%)$ and had a monthly family income of U\$711.3. Most (53.3\%) had DM diagnosis for more than 19 years; Wagner classification 2 $(46.7 \%)$; neuropathic ulcer $(76.7 \%)$; reported having foot ulcer for more than 3 months (73.3\%) and some presented their first episode of foot ulcer $(43.3 \%)$ (Table 1$)$.

Table 1 - Distribution and sociodemographic characterization of the participants in the Patient Testing Step $(n=30)$. Sorocaba, Brazil, 2017.

\begin{tabular}{lllll}
\hline Variables & N & \% & Mean (SD*) & Range \\
\hline Age & & & $57.4(10,7)$ & $35-75$ \\
Race/color & 26 & 86.7 & & \\
White & 4 & 13.3 & & \\
Black/Brown & & & & \\
Sex & 23 & 76.7 & & \\
Male & 7 & 23.3 & & \\
Female & & & & \\
Family arrangement & 5 & 16.7 & & \\
Living alone & 10 & 33.3 & & \\
Living with companion & 10 & 33.3 & & \\
Living with companion and sons & 4 & 13.3 & & \\
Living with sons & 1 & 3.33 & & \\
Other & & & $7.8(12.3)$ & \\
Schooling & & & $71.3(522.4)$ & $179.6-2994.0$ \\
Family income (US dollars) & & &
\end{tabular}




\begin{tabular}{|c|c|c|c|c|}
\hline Occupation & & & & \\
\hline Active & 8 & 26,6 & & \\
\hline Inactive & 22 & 73,4 & & \\
\hline Ulcer duration (months) & & & $11.5(33.9)$ & $1-192$ \\
\hline Length of DM diagnosis (months) & & & $206(106.7)$ & $12-408$ \\
\hline Wagner Classsification & & & & \\
\hline 0 & 0 & 0 & & \\
\hline 1 & 11 & 36.7 & & \\
\hline 2 & 14 & 46.7 & & \\
\hline 3 & 5 & 16.6 & & \\
\hline 4 & 0 & 0 & & \\
\hline Type of foot ulcer & & & & \\
\hline Neuropathic & 23 & 76.7 & & \\
\hline Neuroischemic & 7 & 23.3 & & \\
\hline Number of foot ulcer episode & & & & \\
\hline First & 13 & 43.3 & & \\
\hline Second & 9 & 30 & & \\
\hline Third & 3 & 10 & & \\
\hline$>3$ & 5 & 16.7 & & \\
\hline
\end{tabular}

Regarding the practicability of the Brazilian version of the DFS-SF, evaluated in the Patient Testing Step $(n=30)$, the results suggest that it is an easy-to-apply instrument, averaging $7 \mathrm{~min}$ and $35 \mathrm{sec}$ for application. As for acceptability, data indicate that the questionnaire was easy to understand, since $100 \%$ of the patients answered all the items.

The cognitive debriefing revealed that the participants understood what was questioned in each item of the DFS-SF. In this step, most of the patients did not have difficulty in understanding the questionnaire. Occasionally, they had trouble in understanding or not having clarity of the terms "hobbie", "Not being able to support the foot in the ground" and also the word "Exhaustion".

\section{DISCUSSION}

This study was aimed at providing a validated version of DFS-SF for the Brazilian population, through the accomplishment of the process of linguistic validation of the questionnaire.

Evaluating the QoL of patients with DFU requires an individual understanding focused on treatment guidance, management of complications and patient-health professional relationship ${ }^{12}$. Therefore, the DFS-SF emerged as a tool for this care.

Through the current study, the DFS-SF was linguistic validated and proved to be a valid method for diabetic foot evaluation. The process of culturally adapting an existing instrument rather than developing a new one to a target population is considered advantageous, as it allows the comparison of data obtained from different samples and from different contexts, leading to a more comprehensive assessment of the real situation ${ }^{15}$.

The process of linguistic validation of DFS-SF was carried out according to the methodological standards recommended by internationally recognized publications ${ }^{14}$.

The cultural adaptation of a questionnaire for use in a new country, culture or language re- 
quires a unique methodology in order to achieve equivalence between the original source and the target language. Therefore, the items should not only be translated linguistically, they should be culturally adapted, because the cultural differences of health perception are different in each region, cultural context and lifestyle of the population in question ${ }^{15,20}$.

Regarding the content validity stage, the experts committee maybe considered a pivotal step in order to obtain the intercultural equivalences ${ }^{15}$. An instrument is considered to present evidences of content validity if there is consensus among the experts that it measures what it proposes to measure. In this case, the instruments are submitted to the appreciation of specialists in the area of interest for modification, withdrawal or addition of items ${ }^{21}$. Independently, experts analyze the items, checking their clarity, pertinence, comprehensiveness and evaluating the overall appearance of the instrument. During this step, experts also assess if the items correspond to cultural reality ${ }^{17}$. Thus, eight bilingual experts with experience in cultural adaptation or performance in the theme, research experience involving the application of measuring instruments and the ability to recognize expressions in the English language and Portuguese language participated in this committee.

From this perspective, the experts' suggestions were essential to adjust the English version of the questionnaire for use in the Brazilian scenario. All recommendations were followed and changes were made to the items, even if they presented a satisfactory Content Validity Index, but if suggestions allowed to clarify the questions of the instrument, resulting in an easier tool to understand and apply.

Thus, the expert committee analyzed the semantic-idiomatic, conceptual and cultural equivalences and the questionnaire was evaluated as equivalent, having undergone minor adjustments. It is possible to observe that, although they were few, the semantic and idiomatic equivalence between the original DFS-SF version and its translated version were the ones that had the majority of disagreements.

Thereby, it was possible to maintain the linguistic and cultural equivalence of the items of the original questionnaire, maintaining its relevance even if it is necessary to modify it for the culture of a specific place, as recommended(15). Since most CVIs were higher than 0.80 , there was no need of major alteration or exclusion of any item and all were considered pertinent to the Brazilian culture.

The Brazilian version of DSF-SF was patient-tested among 30 subjects in order to evaluate its practicability and acceptability, presenting a short application time and easy comprehension by the target population - patients with DM. The questionnaire proved to be linguistically validated into the Brazilian culture and, secondly, besides being a comprehensive instrument that evaluates the QoL of people with DF, it can also help nurses and other health professionals outlining tailored interventions aimed at improving it ${ }^{12,13}$.

Another study has shown that the Brazilian version of theDiabetic Foot Ulcer Scale-short form (DFS-SF)presentedgood evidence of reliability, shown by adequate internal consistency(Cronbach's alpha in domains $>0.70)$ and compound reliability(0.84 > CC > 0.92); and of convergent validity, by significant positive correlationsof moderate to strong magnitude with SF-36. Structural construct validitywas examined by applying the DFS-SF confirmatory factor analysis, whichindicated that the Brazilian version of the instrument is properly fitted to theoriginal dimensional structure (22).

Our study has a limitation the sample in which the measurement test was conducted was restricted to people with DF disease from a single institution, which limits the generalization of results. Brazil is a large country with different linguistic influences between different regions. Also, self-report questionnaires may be influenced by the patient's or family's desire for improvement. The questionnaires were applied through interviews due to the patient's difficulties in auto filling them. In addition, the validity of DFS-SF should be tested against other scenarios.

\section{CONCLUSION}

The linguistic validation process of the Brazilian version of the DFS-SF have been com- 
pleted in accordance with internationally recommended standards. The instrument was easy to apply, understand and presented short time for administration. Further studies are being developed in order to assess its factorial structure, reliability and internal validity and allow its use in clinical practice.

\section{BIBLIOGRAPHIC REFERENCES}

The International working Group on the Diabetic Foot. (2015). Guidance on the management and prevention of foot problems in diabetes 2015 .Definitions \& criteria 2015. Retrieved from http://iwgdf.org/guidelines/definitions-criteria- 2015/

Armstrong DS, Andrew JM, Boulton MD. Diabetic foot ulcers and their recurrence. N Engl J Med. 2017;376(24):23672375.

Huang $Y$, Ting $X$, Minjie W, Yemin C, Xiqiao W, Yuzhi J et al. The Investigation of Demographic Characteristics and the Health-Related Quality of Life in Patients With Diabetic Foot Ulcers at First Presentation. Int J Low Extrem Wounds.2012;11 (3):187-93.

Jupiter DC, Thorud JC, Buckley CJ, Shibuya N. The impact of foot ulceration and amputation on mortality in diabetic patients. I: From ulceration to death, a systematic review. Int Wound J. 2016 Oct;13(5):892-903. doi: 10.1111/iwj.12404

Ferreira V, Martins J, Loureiro L, Loureiro T, Borges L, Silveira $D$, et al . Consulta multidisciplinar do pé diabético: avaliação dos fatores de mau prognóstico. Angiol Cir Vasc [Internet]. 2014 Set [citado 2020 Jan 14]; 10( 3 ): 146-150.

Sinwar PD. The diabetic foot management - Recent advance. Int J Surg. 2015 Mar;15:27-30. doi: 10.1016/j. ijsu.2015.01.023

Fejfarová V, Jirkovská A, Dragomirecká E, Game F, Bém $R$, Dubský $M$, et al. Does the diabetic foot have a significant impact on selected psychological or social characteristics of patients with diabetes mellitus? J Diabetes Res. 2014;2014:371938.

Pinilla AE, Barrera MDP, Sánchez AL, Mejía A. Factores de riesgo en diabetes mellitus y pie diabético: un enfoque hacia la prevención primaria. Rev Colombiana de Cardiología. 2013;20(4):213-22.

Brasil. Ministério da Saúde. Secretaria de Atenção à Saúde. Departamento de Atenção Básica. Estratégias para o cuidado da pessoa com doença crônica: diabetes mellitus. Brasília, DF: Ministério da Saúde; 2013.
Bakker K, Apelqvist J, Schaper NC. International Working Group on Diabetic Foot Editorial B. Practical guidelines on the management and prevention of the diabetic foot 2011. Diabetes Metab Res Ver. 2012;28(1):225-31. doi: 10.1002/dmrr.2253.

Almeida SA, Silveira MM, Espírito Santo PF, Pereira RC, Salomé GM. Assessment of the quality of life of patients with diabetes mellitus and foot ulcers. Rev. Bras. Cir. Plást. [Internet]. 2013 Mar [cited 2020 Jan 14] ; 28( 1 ): 142-146.

Abetz L, Sutton M, Brady L, McNulty P, Gagnon DD. The Diabetic Foot Ulcer Scale (DFS): a quality of life instrument for use in clinical trials. Pract Diab Int. 2002;19(6):6775. doi.org/10.1002/pdi.356

Bann CM, Fehnel SE, Gagnon DD. Development and validation of the Diabetic Foot Ulcer Scale - Short Form (DFS-SF). Pharmacoeconomics. 2003;21(17):1277-90.

Mapi Research Trust. (2016). Linguistic Validation Guidance of a Clinical Outcome Assessment (COA). Lyon, France: Mapi.

Beaton D, Bombardier C, Guillemin F, Ferraz MB. Recommendations for the Cross-Cultural Adaptation of the DASH \& QuickDASH Outcome Measure [online]. Intitute for Work and Health; 2007 [acesso em 01 jan 2016]. Disponível em: http://www.dash.iwh.on.ca/system/files/XCulturaIAdaptation-2007.pdf

Hui LF, Yee-Tak Fong D, Yam M, Yuk Ip W. Translation and validation of the Chinese diabetic foot ulcer scale - short form. Patient 2008;1(2):137-45.

Alexandre NMC, Coluci MZO. Validade de conteúdo nos processos de construção e adaptação de instrumentos de medida. Ciênc. saúde coletiva 2011;16(7):3061-8.

Polit DF, Beck CT. Fundamentos de Pesquisa em enfermagem: avaliação de evidências para as práticas da enfermagem. 7.ed. Porto Alegre:Artmed; 2011.

Aaronson N, Alonso J, Burnam A, Lohr KN, Patrick DL, Perrin $E$, et al. Assessing health status and quality-of-life instruments: attributes and review criteria. Qual Life Res. 2002;11(3):193-205.

Mokkink LB, Terwee CB, Knol DL, Stratford PW, Alonso J, Patrick DL, et al. The COSMIN checklist for assessing the methodological quality of studies on measurement properties of health status measurement instruments: an international Delphi study. Qual Life Res. 2010;19(4):539-49

Mcdowell I, Newell C. Measuring Health. A guide to rating scales and questionnaire. 2ed. New York: Oxford; 1996.

de Oliveira Kaizer UA, Alexandre NMC, Rodrigues RCM, Cornélio ME, de Melo Lima MH, São-João TM. Measurement properties and factor analysis of the Diabetic Foot UIcer Scale-short form (DFS-SF). Int Wound J. 2020 Jun;17(3):670-682. doi: 10.1111/iwj.13310. Epub 2020 Feb 17. PMID: 32065735. 


\section{Acknowledgments}

The authors would like to thank the staff of the Wound and Diabetic Foot Ulcer Clinic and all the participants for their kind contribution in data collection stage.

\section{Author contributions}

UAOK designed the study, collected the data, analyzed the data and drafted the manuscript; TMSJ contributed in the design phase, participated in the analysis and made major reviews in the manuscript. NMCA, RCMR, MEC and MHML contributed with critical reviews to the manuscript. All authors read and approved the final manuscript.

Autor correspondente:

Uiara Aline de Oliveira Kaizer

uiara_oliveira@hotmail.com

Editor:

Prof. Dr. Marcelo Riberto

Received in: jul 30, 2020

Approved in: dec 18,2020 